\title{
COMPUTATIONS OF VOLUMES AND EHRHART SERIES IN FOUR CANDIDATES ELECTIONS
}

\author{
WINFRIED BRUNS, BOGDAN ICHIM, AND CHRISTOF SÖGER
}

\begin{abstract}
We describe several experimental results obtained in four candidates social choice elections. These include the Condorcet and Borda paradoxes, as well as the Condorcet efficiency of plurality voting with runoff. The computations are done by Normaliz. It finds precise probabilities as volumes of polytopes and counting functions encoded as Ehrhart series of polytopes.
\end{abstract}

\section{INTRODUCTION}

In [23, p. 382] Lepelley, Louichi and Smaoui state:

Consequently, it is not possible to analyze four candidate elections, where the total number of variables (possible preference rankings) is 24 . We hope that further developments of these algorithms will enable the overcoming of this difficulty.

Normaliz [12] is a software tool that (in particular) may be used for the computation of volumes and Ehrhart series of rational polytopes. In the 18 years of its existence it has found numerous applications. For these, as well for its connections to several computer algebra systems see [12]. One of the driving forces for the improvements of Normaliz in the past years was the desire to solve the problems raised by Schürmann [24], that is to compute the volumes and Ehrhart series of certain polytopes related to social choice.

We believe that the recent development in the algorithms of Normaliz and its offspring NmzIntegrate have (partially) solved the problem raised by Lepelley, Louichi and Smaoui. In [8, p. 388] Brandt, Geist and Strobel write:

To the best of our knowledge, Normaliz is the only program which is able to compute polytopes corresponding to elections with up to four alternatives.

In this paper we present several results of our own computational experiments with four candidates elections. The background of these experiments, motivated by social choice theory and its connection with the polytope theory, may be found in Gehrlein and Lepelley [21], Lepelley, Louichi and Smaoui, [23], Schürmann [24] or Wilson and Pritchard [25]. For discrete convex geometry we refer the reader to Bruns and Gubeladze [9].

As an introductory example we discuss the Condorcet paradox whose probability of $331 / 2048 \approx 16.2 \%$ for four candidates has been known for quite a while (Gehrlein [19]). We continue with the Condorcet efficiency of plurality voting (see [24]) and complement

2010 Mathematics Subject Classification. 52B20, 91B12.

Key words and phrases. rational polytope, volume, Ehrhart series, social choice, Condorcet paradox, Borda paradox. 
it by the Condorcet efficiency of plurality elections with cutoff. While plurality voting has Condorcet efficiency $74.3 \%$, a runoff ballot of two candidates increases it to $91.2 \%$. The gain is substantial and justifies runoff ballots that are part of many voting procedures. (In this introduction we content ourselves with approximate probabilities; precise rational numbers will be given later on.)

Another problem discussed in [24] is "plurality versus runoff", namely the probability of $75.5 \%$ that the plurality winner also wins the runoff.

Next follows a discussion of the four types of antisymmetric relations between four candidates that can arise from comparisons in majority, and their probabilities. As we will see, the case (i) of a linear order is the most likely one by far. The other 3 cases, namely that (ii) there exists a Condorcet winner, but not a loser, (iii) a loser, but not a winner, and (iv) neither a winner, nor a loser, have small probabilities $<10 \%$.

We conclude the list of voting outcomes with the Borda paradoxes. The strict Borda paradox occurs if the outcome of the pairwise majority comparison is a linear order and the plurality outcome completely reverses it. For four candidates its conditional probability is approximately $0.156 \%$. The strong Borda paradox has two variants, namely (i) that the Condorcet loser wins the plurality, and (ii) that the Condorcet winner is the last in plurality. As to be expected, their conditional probabilities are considerably larger, namely about $2.268 \%$ and $2.379 \%$. Though one would intuitively expect that these probabilities agree, they are not equal.

All these events are discussed together with their defining inequalities in Section2, We would like to point out that Normaliz not only computes the volumes of the polytopes in all cases, but also their Ehrhart series, and therefore precise numbers of election results that represent the events under discussion, depending on the number of voters. We give the complete numerics only for the Condordet paradox, but all data can be obtained by request from the authors.

Ehrhart series (see Section 3) are more easily to compute for closed polytopes than for the ones that arise if one excludes ties. However, in many cases the Ehrhart series of the semiopen polytope can be computed from that of its closure (and conversely). The crucial condition is that all inequalities, except the sign conditions, defining the semiopen polytope are strict, and they are satisfied with equality by the election result in which every preference order has the same number of voters. This follows from a variant of Ehrhart reciprocity (for example, see [9, Th. 6.51]).

Normaliz can do all computations in dimension 24 (the number of preference orders for four candidates), but the the computation times range from seconds (for the Condorcet paradox) to a few days (for the Condorcet efficiency of the runoff scheme) on a fast machine that allows 32 parallel threads. Schürmann [24] made the elegant observation that many computations can be enormously accelerated if one uses the symmetry that is inherent in many polytopes. Only two of the polytopes discussed in this paper do not allow this approach, namely linear order and (consequently) the strict Borda paradox. Symmetrization, which requires the computation of weighted Ehrhart series, shrinks computation times from days to hours, minutes, or even tenths of seconds, depending on the example. Symmetrization is discussed in Section 4, and all relevant computation data are listed in Section 5 . 
The reader who is interested in a deeper understanding of the mathematics and algorithms of Normaliz and NmzIntegrate is refereed to the articles by Bruns with Koch [14], Ichim [11], Hemmecke, Ichim, Köppe and Söger[10], Ichim and Söger [13], Söger [15] and Sieg and Söger [16].

\section{POLYTOPES IN FOUR CANDIDATES ELECTIONS AND THEIR VOLUMES}

2.1. Voting schemes and volumes of rational polytopes. We briefly sketch the connection between rational polytopes and social choice, referring the reader to [21], [23], [24] or [25] for details and a more extensive treatment. As an introductory example we first consider the well-known Condorcet paradox. The polytopes $\mathcal{P}$ associated to it and to other voting events are semiopen: $\mathcal{P}$ is the bounded set of solutions of a system of linear equations and inequalities in which some of the inequalities may be strict.

Consider an election in which each of the $k$ voters fixes a linear preference order of $n$ candidates. In other words, voter $i$ chooses a linear order $j_{1} \succ_{i} \cdots \succ_{i} j_{n}$ of the candidates $1, \ldots, n$. There are $N=n$ ! such linear orders that we list lexicographically. The election result is the $N$-tuple $\left(v_{1}, \ldots, v_{N}\right)$ in which $v_{p}$ is the number of voters that have chosen the preference order $p$. Then $v_{1}+\cdots+v_{N}=k$, and $\left(v_{1}, \ldots, v_{N}\right)$ can be considered as a lattice point in the positive orthant $\mathbb{R}_{+}^{N}$ of $\mathbb{R}^{B}$, more precisely, as a lattice point in the simplex

$$
\mathcal{U}_{k}^{(n)}=\mathbb{R}_{+}^{N} \cap A_{k}=k\left(\mathbb{R}_{+}^{N} \cap A_{1}\right)=k \mathcal{U}^{(n)}
$$

where $A_{k}$ is the hyperplane defined by $v_{1}+\cdots+v_{N}=k$, and $\mathcal{U}^{(n)}=\mathcal{U}_{1}^{(n)}$ is the unit simplex of dimension $N-1$ naturally embedded in $N$-space. $\left(U^{(n)}\right.$ is the convex hull of the unit vectors; see Figure 1,) The further discussion is based on the Impartial Anonymous

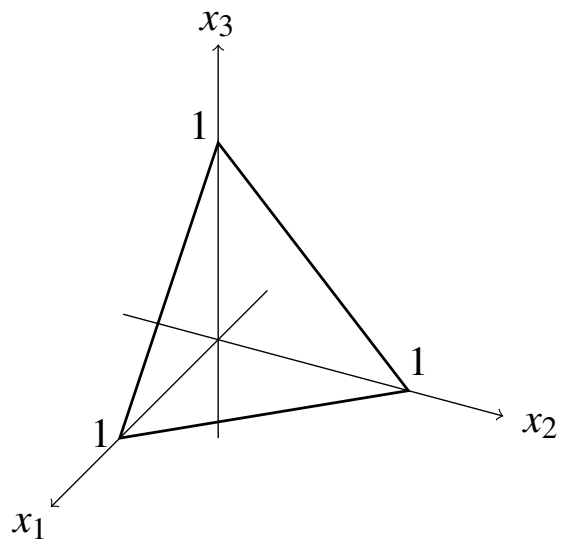

FIGURE 1. The unit simplex in $\mathbb{R}^{3}$

Culture assumption that all lattice points in the simplex $\mathcal{U}_{k}^{(n)}$ have equal probability of being the outcome of the election.

We fix a specific outcome $v=\left(v_{1}, \ldots, v_{N}\right)$. By the majority rule candidate $j$ beats candidate $j^{\prime}$ in the election with result $v$ if

$$
\#\left\{i: j \succ_{i} j^{\prime}: i=1, \ldots, k\right\}>\#\left\{i: j^{\prime} \succ_{i} j: i=1, \ldots, k\right\} .
$$


As the Marquis de Condorcet [17] observed, the relation "beats" is nontransitive in general, and one must ask for the probability of Condorcet's paradoxon, namely an outcome without a Condorcet winner, where candidate $j$ is a Condorcet winner if $j$ beats all other candidates $j^{\prime}$. The Condorcet winner will sometimes be denoted $\mathrm{CW}$ in the following. Given the number $k$ of voters, let $p_{\mathrm{CW}}^{(n, j)}(k)$ denote the probability that candidate $j$ is the Condorcet winner, and $p_{\mathrm{CW}}^{(n)}(k)$ the probability that there is a Condorcet winner at all. By symmetry and by mutual exclusion, $p_{\mathrm{CW}}^{(n)}(k)=n \cdot p_{\mathrm{CW}}^{(n, j)}(k)$.

Now, if we assume that the number $k$ of voters is very large, then we are mainly interested in the limit

$$
p_{\mathrm{CW}}^{(n)}=\lim _{k \rightarrow \infty} p_{\mathrm{CW}}^{(n)}(k)=n \lim _{k \rightarrow \infty} p_{\mathrm{CW}}^{(n, j)}(k)=n \cdot p_{\mathrm{CW}}^{(n, j)} .
$$

Let us fix candidate 1. It is not hard to see that the $n-1$ inequalities (2.1) for $j=1$ and $j^{\prime}=2, \ldots, n$ constitute homogeneous linear inequalities in the variables $v_{1}, \ldots, v_{N}$. Together with the sign conditions $v_{i} \geq 0$ they define a semi-open subpolytope $\mathrm{C}_{k}^{(n)}$ of $\mathcal{U}_{k}^{(n)}$. Then

$$
p_{\mathrm{CW}}^{(n, 1)}=\lim _{k \rightarrow \infty} \frac{\#\left(\mathcal{C}_{k}^{(n)} \cap \mathbb{Z}^{N}\right)}{\#\left(\mathcal{U}_{k}^{(n)} \cap \mathbb{Z}^{N}\right)}=\frac{\operatorname{vol} \mathcal{C}_{1}^{(n)}}{\operatorname{vol} \mathcal{U}_{1}^{(n)}}=\operatorname{vol} \bar{\complement}^{(n)}
$$

where ${ }^{-}$denotes closure and $\mathrm{C}^{(n)}=\mathrm{C}_{1}^{(n)}$. For the validity of (2.2) note that we work with the lattice normalized volume in which the unit simplex has volume 1.

In the case of two candidates, Concordet's paradox cannot occur (if one excludes draws), and for three candidates the relevant volume is not hard to compute, even without a computer (see Gehrlein and Fishburn [20]).

The situation changes significantly for four candidates since $\mathcal{C}^{(4)}$ has dimension 23 and 234 vertices. From now on we will simplify our notation and omit often the superscript (4) when dealing with four candidates. As a subpolytope of $\mathcal{U}, \mathcal{C}$ is cut out by the inequalities $\lambda_{i}(v)>0, i=1,2,3$ whose coefficients are in the first 3 rows displayed in Table 1, For the assignment of indices the preference orders are listed lexicographically, starting with $1 \succ 2 \succ 3 \succ 4$ and ending with $4 \succ 3 \succ 2 \succ 1$.

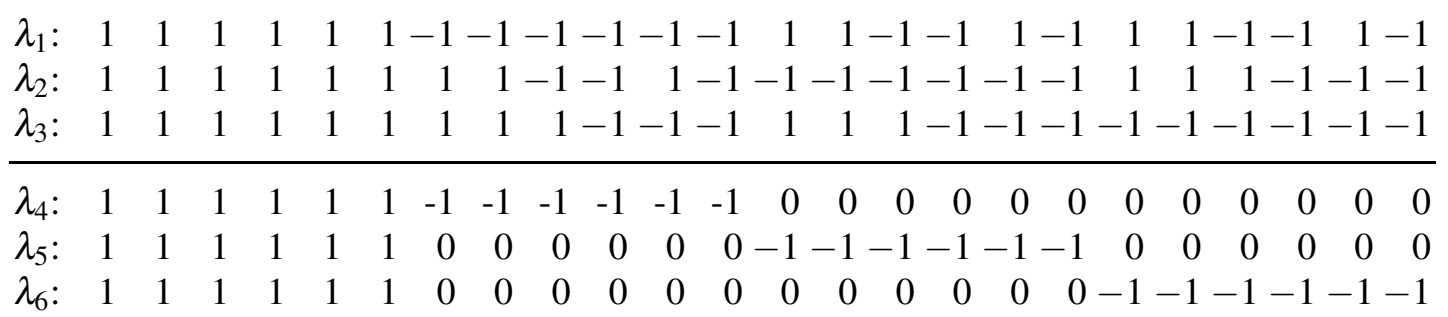

TABLE 1. Inequalities for $\mathcal{C}$ and $\mathcal{E}$

Normaliz computes

$$
\operatorname{vol} \mathcal{C}=\frac{1717}{8192}
$$


in a few seconds. (The combinatorial data of all polytopes discussed and the computation times are listed in Section 5.) It follows that $p_{\mathrm{CW}}=1717 / 2048 \approx 0.8384$. This value was first determined by Gehrlein in [19].

2.2. Plurality rule and runoff. The simplest way out of the dilemma that there may not exist a Condorcet winner is plurality voting: candidate $j$ is the plurality winner if $j$ has more first places in the preference orders of the voters than any of the other $n-1$ candidates. A problem discussed in [24] is plurality voting versus plurality runoff. It goes as follows. In the first round of the election the two top candidates in plurality are selected, and in the second round the preference orders are restricted to these two candidates. In order to model this situation by inequalities one must fix an outcome of the first round of plurality voting, for example we may assume that candidate 1 is the winner of the first round and candidate 2 is placed second after the first round. The chosen outcome gives rise to $n-1$ inequalities. Then the $n$-th inequality expresses that 1 is also the winner of the second round. The volume of the corresponding polytope gives the probability of this event. By mutual exclusion and symmetry, we must multiply the volume by $n(n-1)$ in order to obtain the probability for the event that the winner of the first plurality round wins after runoff.

As a subpolytope of $\mathcal{U}$, the polytope $\mathcal{Q}$ is defined by the inequalities in Table 2 , The

$\begin{array}{rrrrllrrrrrrrrrrrrrrrrrr}1 & 1 & 1 & 1 & 1 & 1 & -1 & -1 & -1 & -1 & -1 & -1 & 0 & 0 & 0 & 0 & 0 & 0 & 0 & 0 & 0 & 0 & 0 & 0 \\ 0 & 0 & 0 & 0 & 0 & 0 & 1 & 1 & 1 & 1 & 1 & 1 & -1 & -1 & -1 & -1 & -1 & -1 & 0 & 0 & 0 & 0 & 0 & 0 \\ 0 & 0 & 0 & 0 & 0 & 0 & 1 & 1 & 1 & 1 & 1 & 1 & 0 & 0 & 0 & 0 & 0 & 0 & -1 & -1 & -1 & -1 & -1 & -1 \\ 1 & 1 & 1 & 1 & 1 & 1 & -1 & -1 & -1 & -1 & -1 & -1 & 1 & 1 & -1 & -1 & 1 & -1 & 1 & 1 & -1 & -1 & 1 & -1\end{array}$

TABLE 2. Inequalities for $Q$

volume is

$$
\operatorname{vol} \mathcal{Q}=\frac{9185069468583833}{146081389744226304}
$$

The total probability of the event that the winner of the first plurality round wins after runoff is

$$
12 \cdot \operatorname{vol} Q=\frac{9185069468583833}{12173449145352192} \approx 0.7545
$$

Therefore, the probability of the failure of the winner of the first round to win the runoff is

$$
1-\frac{9185069468583833}{12173449145352192} \approx 0.2455
$$

in accordance with the results of [24] for a similar model. This computation was first performed by De Loera, Dutra, Köppe, Moreinis, Pinto and Wu in [18], where LattE Integrale [4] was used for the volume computation.

2.3. Condorcet efficiency. The last problem discussed in [24] is the Condorcet efficiency of plurality voting. It is the conditional probability that the Condorcet winner, provided that such exists, is elected by plurality voting, as $k \rightarrow \infty$. (Similarly one defines the Condorcet efficiency of other voting schemes.) Therefore one must compute the probability of the event that candidate $j$ is both the Condorcet winner and the plurality winner. By 
symmetry, one can assume $j=1$. The semi-open polytope $\varepsilon_{k}^{(n)}$, whose lattice points represent this expected outcome, is cut out from $\mathcal{C}_{k}^{(n)}$ by $n-1$ further inequalities saying that 1 has more first places than any of the other $n-1$ candidates. Thus one obtains

$$
\frac{n \operatorname{vol} \mathcal{E}^{(n)}}{p_{\mathrm{CW}}^{(n)}}
$$

as the Condorcet efficiency of plurality voting where $\mathcal{E}^{(n)}=\mathcal{E}_{1}^{(n)}$.

The extra 3 inequalities $\lambda_{i}(v)>0, i=4,5,6$, given in the last 3 lines of Table 1 increase the complexity of the polytope $\mathcal{E}$ enormously in comparison to $\mathcal{C}$. Nevertheless, Normaliz computes the volume in moderate time. We have obtained

$$
\operatorname{vol} \mathcal{E}=\frac{10658098255011916449318509}{68475651442606080000000000}
$$

so that the Condorcet efficiency of plurality voting turns out to be

$$
\frac{4 \mathrm{vol} \varepsilon}{p_{\mathrm{CW}}}=\frac{10658098255011916449318509}{14352135440302080000000000} \approx 0.7426,
$$

in perfect accordance with [24].

Remark 1. Schürmann [24] used variants of the polytopes $Q$ and $\mathcal{E}$. Our choices (which are demanding slightly more computational resources) avoid inclusion-exclusion calculations that would come up in Section 3 .

It is interesting to compare the Condorcet efficiency of plurality voting to the the Condorcet efficiency of the runoff voting scheme. In other words, given that there exists a Condorcet winner, what is the probability that she or he is at least second in plurality?

As above, let us assume that candidate 1 is the Condorcet winner. Then there are $n$ possible cases. The first case is when candidate 1 is the plurality winner as well, and this case was studied above. The other $n-1$ cases are associated to the event that candidate $j$ wins or ties candidate 1 in plurality (where $j \neq 1$ ), while candidate 1 wins the plurality voting against all other candidates. By symmetry, these $n-1$ cases are identical and yield the same volume. The $n$ cases are mutually disjoint and exhaust all the possibilities. (Disjointness is important in Section 3 for avoiding complicated inclusion-exclusion calculations.) The semi-open polytope $\mathcal{F}_{1, j}^{(n)}$, whose lattice points represent the outcome of the cases $j=2, \ldots, n$, is cut out from $\mathrm{C}_{k}^{(n)}$ by the closed condition that candidate $j$ wins against or ties with candidate 1 in plurality and by $n-2$ inequalities saying that 1 has more first places than the remaining $n-2$ candidates. Thus one obtains

$$
\frac{n \operatorname{vol} \mathcal{E}^{(n)}+n(n-1) \operatorname{vol} \mathcal{F}^{(n)}}{p_{\mathrm{CW}}^{(n)}}
$$

as the the Condorcet efficiency of the runoff, where $\mathcal{F}^{(n)}=\mathcal{F}_{1,2}^{(n)}$.

As a subpolytope of $\mathcal{U}$, the polytope $\mathcal{F}$ is defined by the inequalities in Table 3 , where the last line should be interpreted as $\overline{-\lambda_{4}}(v) \geq 0$, expressing the condition that candidate 1 does not beat candidate 2 in plurality. 


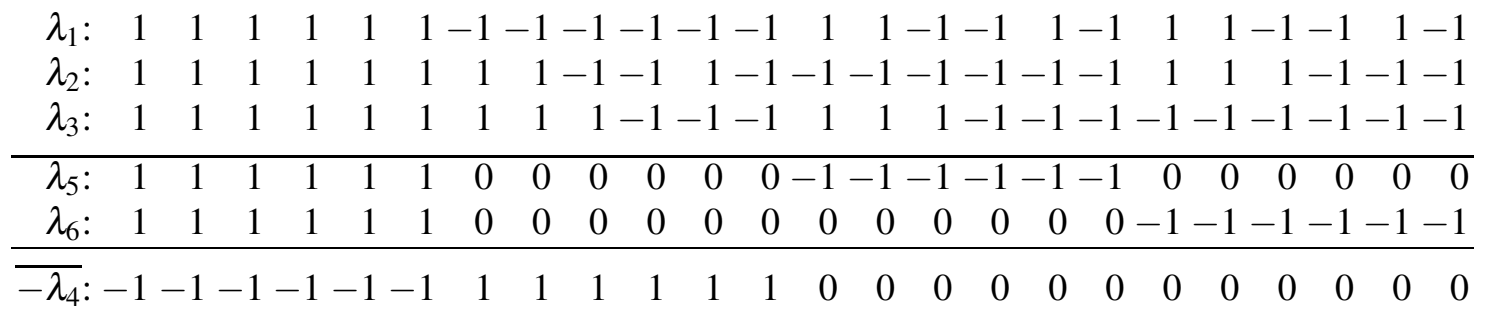

TABLE 3. Inequalities for $\mathcal{F}$

The volume is

$$
\operatorname{vol} \mathcal{F}=\frac{7280153240719060220104571}{616280862983454720000000000} .
$$

Finally, the Condorcet efficiency of the runoff is

$$
\frac{4 \operatorname{vol} \mathcal{E}+12 \operatorname{vol} \mathcal{F}}{p_{\mathrm{CW}}}=\frac{19627224002877404784030049}{21528203160453120000000000} \approx 0.9117 .
$$

2.4. Condorcet classification. In this subsection we classify the asymmetric relations between four candidates given by the majority rule. To the best of our knowledge the computations of the probabilities of the classes is new.

Let us first outline a duality argument that will be used several times below. Consider an election result $v=\left(v_{1}, \ldots, v_{N}\right)$ for $n$ candidates, where $N=n !$, the $N$ preference orders $\pi_{1}, \ldots, \pi_{N}$ are listed in some order, and $v_{i}$ is the number of voters of $\pi_{i}$. Each preference order has an inverse $c(\pi)$ that ranks the candidates in inverse order relative to $\pi$ : the inverse order to $1 \succ 2 \succ 3 \succ 4$ is $4 \succ 3 \succ 2 \succ 1$ etc. The assignment $\pi \rightarrow c(\pi)$ defines a permutation of the sequence $1, \ldots, N$, sending $i$ to the index of $c\left(\pi_{i}\right)$. The induced permutation of the coordinates of $\mathbb{R}^{N}$ is called inversion of preference orders. It inverts all comparisons in majority. In particular it turns a Condorcet winner into a Condorcet loser, and conversely.

The results of the $n$ candidates elections may be classified in two main categories:

(A) There exists a Condorcet winner. As seen above, in the case of four candidates elections the results fall into this category with probability

$$
P(\exists \text { Condorcet Winner })=1717 / 2048 \approx 0.8384 \text {. }
$$

(B) There exists no Condorcet winner. For four candidates elections the results fall into this category with probability

$$
P(\nexists \text { Condorcet Winner })=1-1717 / 2048=331 / 2048 \approx 0.1616 \text {. }
$$

We refer the reader to Gehrlein and Lepelley [21, Section 3.2.1] or [22] for a discussion of the three candidates situation. Note that in the three candidates scenario the event that there exist a linear order on the result of the majority voting is the same as the event that there exists a Condorcet winner, since the other two candidates are automatically ordered. This no longer true for four or more candidates. Even if a Condorcet winner exists, the remaining candidates need not to be linearly ordered. Therefore, we need to further refine our classification.

We discuss the case of four candidates in detail: 
(A) Assume that a Condorcet winner (CW) does exist. This situation must be split into two subcategories:

(1) The result of the majority voting defines a linear order of the candidates. This further implies (independently of the number of candidates $n$ ) that there also exists a Condorcet loser (CL).

(2) There exist no linear order on the result of the majority voting. In the (particular) case of four candidates elections this is equivalent to saying that there exists a cycle of length three among the lower candidates (i.e., the three candidates Condorcet Paradox) or that there exists no Condorcet loser.

(B) Now assume the case that a Condorcet winner does not exist. This situation must also be split into two subcategories:

(1) There exists a cycle of order three among the candidates and a Condorcet loser. Inversion of preference orders turns this case into (A2). In particular they have the same probability.

(2) There exist a cycle of length four among the candidates or (equivalently) there exists no Condorcet loser. This condition defines only 4 of the 6 relations between the candidates, but it easy to check that all 4 possibilities for the remaining 2 relations are equivalent up to a renaming of the candidates.
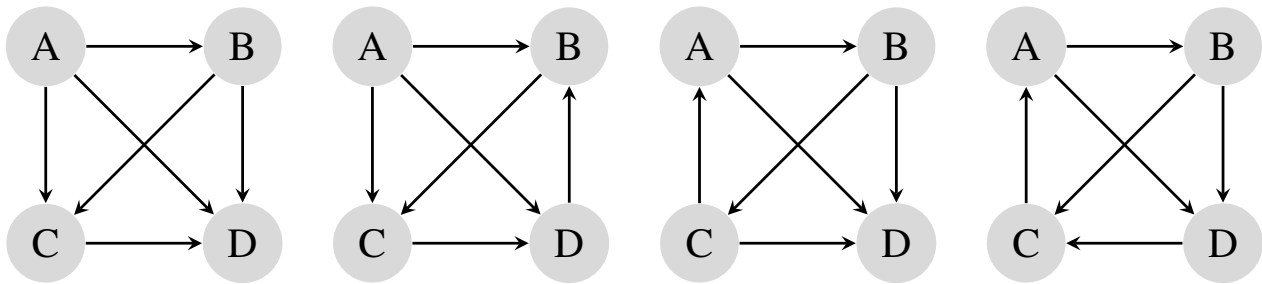

FIGURE 2. Oriented graphs representing the Condorcet classes of four candidates with respect to the relation given by the majority rule

In order to compute the probabilities of the 4 classes, we consider the polytope $\mathcal{T}$ which corresponds to the event that candidate 1 beats candidates $2,3,4$, candidate 2 beats candidates 3,4 and candidate 3 beats candidates 4 . In other words, $\mathcal{T}$ represents the linear order. As a subpolytope of $\mathcal{U}$, the polytope $\mathcal{T}$ is defined by the inequalities in Table 4. (Note that $\beta_{i}=\lambda_{i}$ for $i=1,2,3$.)

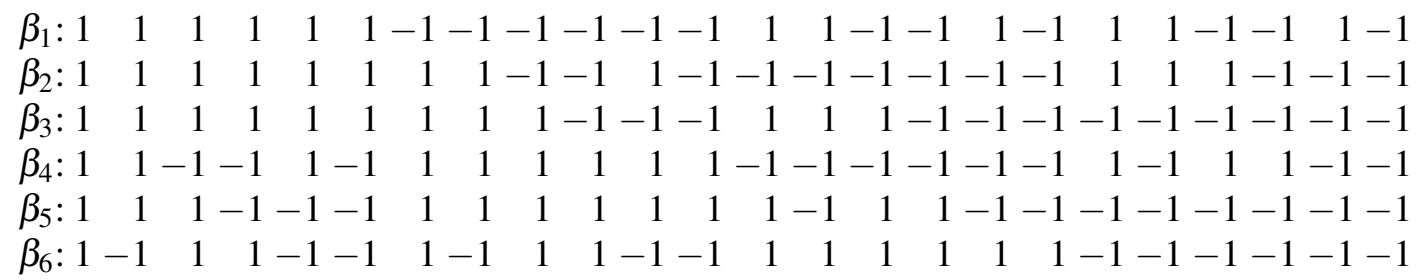

TABLE 4. Inequalities for $\mathcal{T}$

We have obtained

$$
\operatorname{vol} \mathcal{T}=\frac{5507086513}{173946175488}
$$


Since a set of 4 elements admits 24 possible linear orders, the probability to have a linear order on the result of the majority voting is

$$
P(\exists \mathrm{CW}, \exists \mathrm{CL})=\frac{5507086513}{7247757312} \approx 0.7598 .
$$

It follows that the probability that a Condorcet winner does exists, and still there exist no linear order on the result of the majority voting is

$$
P(\exists \mathrm{CW}, \nexists \mathrm{CL})=\frac{1717}{2048}-\frac{5507086513}{7247757312}=\frac{569280335}{7247757312} \approx 0.07855 \text {. }
$$

By the duality argument observed in the case (B1) the probability that a Condorcet loser does exists, but no Condorcet winner, is

$$
P(\nexists \mathrm{CW}, \exists \mathrm{CL})=\frac{569280335}{7247757312} \approx 0.07855
$$

as well. The probability of the remaining class (B2), the existence of a 4-cycle, is

$$
P(\nexists \mathrm{CW}, \nexists \mathrm{CL})=1-\frac{5507086513}{7247757312}-2 * \frac{569280335}{7247757312}=\frac{602110129}{7247757312} \approx 0.0831 \text {. }
$$

As a test for the correctness of the algorithm, we have nevertheless computed the probability of a 4-cycle directly. To this end, we consider the polytope $\mathcal{K}$ corresponding to the event that candidate 1 beats candidates 2 , candidate 2 beats candidates 3 , candidate 3 beats candidates 4 and candidate 4 beats candidates 1 . As a subpolytope of $\mathcal{U}$, the polytope $\mathcal{K}$ is defined by the inequalities in Table 5, We have obtained

$$
\begin{aligned}
& \beta_{1}: \quad 1 \quad 1 \quad 1 \quad 1 \quad 1 \quad 1-1-1-1-1-1-1 \quad 1 \quad 1-1-1 \quad 1-1 \quad 1 \quad 1-1-1 \quad 1-1 \\
& \beta_{4}: \quad 1 \quad 1-1-1 \quad 1-1 \quad 1 \quad 1 \quad 1 \quad 1 \quad 1 \quad 1-1-1-1-1-1-1 \quad 1-1 \quad 1 \quad 1-1-1 \\
& \beta_{6}: 1-1 \quad 1 \quad 1-1-1 \quad 1-1 \quad 1 \quad 1-1-1 \quad 1 \quad 1 \quad 1 \quad 1 \quad 1 \quad 1-1-1-1-1-1-1
\end{aligned}
$$

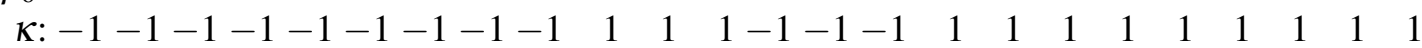

TABLE 5. Inequalities for $\mathcal{K}$

$$
\operatorname{vol} \mathcal{K}=\frac{602110129}{43486543872}
$$

Since a set of 4 elements admits 6 possible cycles of length four among the elements (if we fix one element there are 6 possible linear orders among the remaining three elements), one obtains exactly the same probability for the class (B2) that was computed indirectly above.

2.5. Borda paradoxes. In this subsection we study a family of voting paradoxes, first introduced by the Chevalier de Borda in [7]. To the best of our knowledge all volume computations in this subsection are new.

The strict Borda paradox appears in a voting situation when there is a complete reversal of the ranking of candidates given by the majority voting and plurality voting. In order to model this situation by inequalities one must fist assume that there exist a linear order 
on the result of the majority voting that involves all $n$ candidates, say $1, \ldots, n$ in this order (there are $n$ ! possible outcomes). The chosen outcome gives rise to

$$
\frac{n(n-1)}{2}
$$

inequalities. Then one must add $n-1$ inequalities expressing that the order was completely reversed by the plurality voting. The volume of the corresponding polytope gives the probability of this event. By mutual exclusion and symmetry, we must multiply the volume by $n$ ! and then take the conditional probability under the hypothesis that such a linear order exists.

As a subpolytope of $\mathcal{U}$, the polytope $\mathcal{B}_{\mathrm{St}}$ is defined by the inequalities in Table 6 , Note

$$
\begin{aligned}
& \begin{array}{llllllllllllllllll}
\beta_{1}: & 1 & 1 & 1 & 1 & 1 & 1-1-1-1-1-1-1 & 1 & 1-1-1 & 1-1 & 1 & 1-1-1 & 1-1
\end{array} \\
& \beta_{2}: \quad 1 \quad 1 \quad 1 \quad 1 \quad 1 \quad 1 \quad 1 \quad 1 \quad 1-1-1 \quad 1-1-1-1-1-1-1-1 \quad 1 \quad 1 \quad 1-1-1-1 \\
& \beta_{3}: \quad 1 \quad 1 \quad 1 \quad 1 \quad 1 \quad 1 \quad 1 \quad 1 \quad 1-1-1-1 \quad 1 \quad 1 \quad 1-1-1-1-1-1-1-1-1-1 \\
& \beta_{4}: \quad 1 \quad 1-1-1 \quad 1-1 \quad 1 \quad 1 \quad 1 \quad 1 \quad 1 \quad 1-1-1-1-1-1-1 \quad 1-1 \quad 1 \quad 1-1-1 \\
& \beta_{5}: \quad 1 \quad 1 \quad 1-1-1-1 \quad 1 \quad 1 \quad 1 \quad 1 \quad 1 \quad 1 \quad 1 \quad 1-1 \quad 1 \quad 1-1-1-1-1-1-1-1-1 \\
& \beta_{6}: \quad 1-1 \quad 1 \quad 1-1-1 \quad 1-1 \quad 1 \quad 1-1-1 \quad 1 \quad 1 \quad 1 \quad 1 \quad 1 \quad 1-1-1-1-1-1-1
\end{aligned}
$$

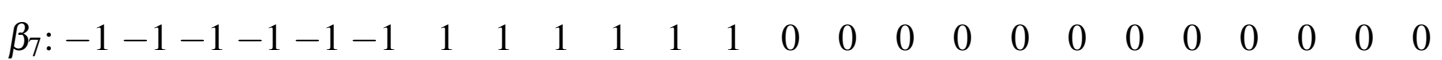

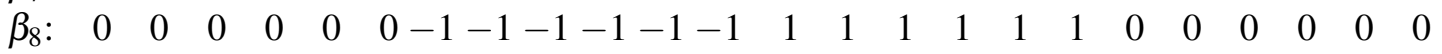

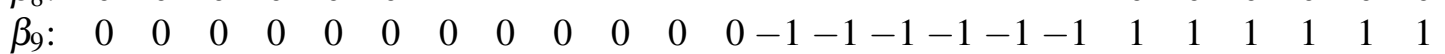

TABLE 6. Inequalities for $\mathcal{B}_{\mathrm{St}}$

that the first six inequalities describe the event that the result of the majority voting yields a linear order. We have obtained

$$
\operatorname{vol} \mathcal{B}_{\mathrm{St}}=\frac{1281727528386311499990911876166511}{25940255058441281524973174784000000000} \text {. }
$$

Finally, conditioned by the assumption that there exists a linear order on the result of the majority voting, we get the probability of the strict Borda paradox for large numbers of voters,

$$
B_{\mathrm{St}}=\frac{24 \cdot \operatorname{vol} \mathcal{B}_{\mathrm{St}}}{24 \cdot \operatorname{vol} \mathcal{T}}=\frac{1281727528386311499990911876166511}{821261107784328041072841984000000000} \approx 0.00156 .
$$

Remark 2. (a) The inequalities defining the strict Borda paradox have an obvious property, which we only state since it does not hold in the case of the strong Borda paradox discussed below: it does not matter if one considers the inequalities in Table 6 or the inequalities defined by the same linear forms multiplied by -1 . In fact, the multiplication by -1 reverses the linear order on the candidates both for majority and plurality, and thus amounts to a renaming of the candidates.

(b) One may ask, as in [22], what happens if the negative plurality rule is used instead of the plurality rule. The negative plurality rule requires the voters to cast a vote against their least preferred candidate. It is not difficult to see that inversion of the preference orders as discussed in subsection 2.4 above, maps an event representing the strict Borda paradox for plurality to an event representing the strict Borda paradox for negative plurality. Therefore no new computation is necessary. 
(c) With the notation used in [22, Formula 19], we have

$$
B_{\mathrm{St}}=P_{\mathrm{StBR}}^{\mathrm{PR}}(4, k, \mathrm{IAC})=P_{\mathrm{StBR}}^{\mathrm{NPR}}(4, k, \mathrm{IAC}) \quad \text { for } k \rightarrow \infty .
$$

We note that the probability of observing the strict Borda paradox in four candidates elections (under the Impartial Anonymous Culture hypothesis) is significantly smaller than the probability of observing the strict Borda paradox in three candidates elections, which was computed in [22, Formula 19] and is $1 / 90 \approx 0.0111$.

The strong Borda paradox is the voting situation in which there is an inversion between the winner or the loser from the majority voting to plurality voting. It appears that de Borda was primarily concerned with the outcome that plurality voting might elect the majority voting loser [7]. In the following we say that the strong Borda paradox occurs if the Condorcet loser is the winner of the plurality voting. Let us assume that candidate 1 is the Condorcet loser. Next, by assuming that 1 wins the plurality we obtain $n-1$ new inequalities. Each candidate may fulfill these conditions, so by symmetry the result must be multiplied by $n$. Finally, the conditional probability has to be computed.

As a subpolytope of $\mathcal{U}$, the polytope $\mathcal{B}_{\mathrm{Sg}}$ is defined by the inequalities in Table 7 , One

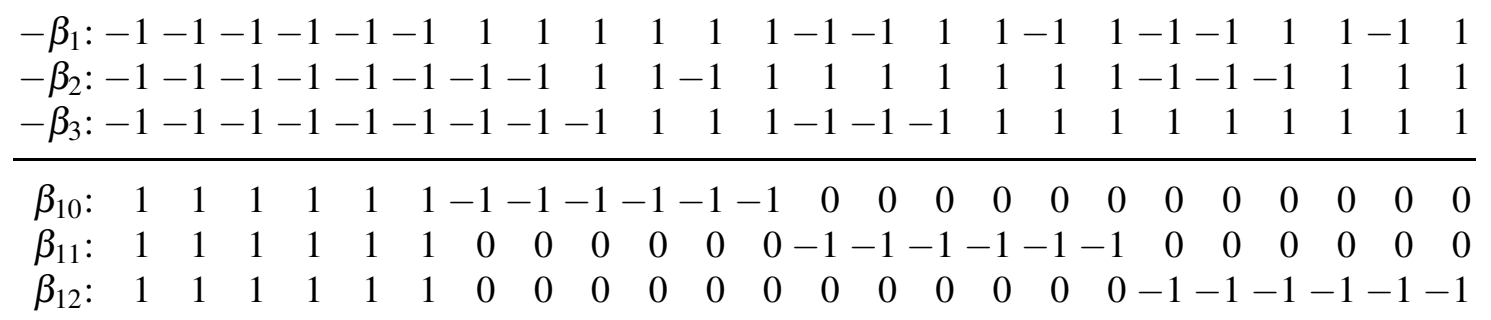

TABLE 7. Inequalities for $\mathcal{B}_{\mathrm{Sg}}$

gets

$$
\operatorname{vol} \mathcal{B}_{\mathrm{Sg}}=\frac{325451674835828550681491}{68475651442606080000000000}
$$

Combined with the previous computations, we obtain the probability of the strong Borda paradox for large numbers of voters:

$$
B_{\mathrm{Sg}}=\frac{4 \cdot \operatorname{vol} \mathcal{B}_{\mathrm{Sg}}}{p_{\mathrm{CW}}}=\frac{325451674835828550681491}{14352135440302080000000000} \approx 0.02268 .
$$

(We have used that the probability that there exists a Condorcet loser equals the probability that there exists a Condorcet winner.)

The situation when the Condorcet winner is the loser of the plurality voting presents also interest, in which case we say that the reverse strong Borda paradox appears. This situation is easy to model: we simply have to multiply the linear forms of Table 7 by -1 . In other words, as a subpolytope of $\mathcal{U}$, the polytope $\mathcal{B}_{\mathrm{SgRev}}$ is defined by the inequalities $\beta_{1}, \beta_{2}, \beta_{4},-\beta_{10},-\beta_{11},-\beta_{12}$. Its volume is given by

$$
\operatorname{vol} \mathcal{B}_{\text {SgRev }}=\frac{104898234852130241}{21035720123168587776} \text {. }
$$


Combined with the previous computations, we get the probability of the reverse strong Borda paradox for large numbers of voters

$$
B_{\mathrm{SgRev}}=\frac{4 \cdot \operatorname{vol} \mathcal{B}_{\mathrm{SgRev}}}{p_{\mathrm{CW}}}=\frac{104898234852130241}{4408976007260798976} \approx 0.02379 .
$$

Though the difference between the two forms of the strong Borda paradox may seem surprising, there are very simple arguments for the asymmetry, independent of any computations. See Remark 8 (c).

Remark 3. (a) Again one may ask what happens if the negative plurality rule (NPR) is used for the strong Borda paradox (BP) and its reverse variant. In total we then have 4 variants. But the 2 new variants are isomorphic to those considered above via the inversion of preference orders:

$$
\text { reverse } \mathrm{BP} \text { with } \mathrm{NPR} \cong \mathrm{BP}, \quad \mathrm{BP} \text { with } \mathrm{NPR} \cong \text { reverse } \mathrm{BP} \text {. }
$$

In fact, the inversion of preference orders turns an event representing the strong Borda paradox for plurality into an event for the reverse strong Borda paradox with negative plurality. Similarly it exchanges the events within the other pair.

(b) With the notation used in [22, Formula 20], we have

$$
\begin{aligned}
B_{\mathrm{Sg}} & =P_{\mathrm{SgBR}}^{\mathrm{PR}}(4, k, \mathrm{IAC}) \quad \text { and } \\
B_{\mathrm{SgRev}} & =P_{\mathrm{SgBR}}^{\mathrm{NPR}}(4, k, \mathrm{IAC}) \quad \text { for } k \rightarrow \infty .
\end{aligned}
$$

We note that the probability of observing the strong Borda paradox in four candidates elections under the plurality rule (respectively the negative plurality rule) is smaller, but still of the same magnitude, with the probability of observing the strong Borda paradox in three candidates elections under the plurality rule (respectively the negative plurality rule), which was computed in [22, Formula 20] and is $4 / 135 \approx 0.0296$ (respectively $17 / 540 \approx$ $0.0315)$.

Remark 4. After the initial submission of this paper to arXiv.org, Lepelley informed us by e-mail that several volume computations can also be done by a combination of the software packages LattE [4] and lrs [3]. He has obtained precisely the same results as we. This is a good test for the correctness of all algorithms involved.

\section{COMPUTATIONS OF EHRHART SERIES AND QUASIPOLYNOMIALS ARISING IN FOUR CANDIDATES ELECTIONS}

While the probability of a certain type of election result, for example the Condorcet paradox, can be computed as the volume of a polytope (or the sum of such volumes), one can use the polyhedral method also to find the exact number of election results of the given type for a specific number $k$ of voters. For example, if $\mathcal{C}$ is the semiopen polytope defined by the condition that candidate 1 is the Condorcet winner, then the number of election results for $k$ voters with Condorcet winner 1 is

$$
E(\mathcal{C}, k)=\#\left(k \mathcal{\cap} \cap \mathbb{Z}_{+}^{N}\right) .
$$


The function $E(\mathcal{C}, k)$ is called the Ehrhart function of $\mathcal{C}$. The best approach to its computations uses the generating function

$$
E_{\mathcal{P}}(t)=\sum_{k=0}^{\infty} E(\mathcal{C}, k) t^{k}
$$

This Ehrhart series is the power series expansion of a rational function at the origin. It is computed as a rational function, and in the following we will always represent Ehrhart series in the form numerator/denominator. The numerator is a polynomial with integer coefficients. The denominator can always be written as a product of $d$ terms $1-t^{g}, d=$ $\operatorname{dim} \mathcal{P}+1$ :

$$
E_{\mathcal{P}}(t)=\frac{h_{0}+h_{1} t+\cdots+h_{s} t^{s}}{\left(1-t^{g_{1}}\right) \cdots\left(1-t^{g_{d}}\right)}, \quad h_{i} \in \mathbb{Z} .
$$

Note that in general there exists no canonical representation in this form; see Bruns, Ichim and Söger [13, Section 4] for a brief discussion of this problem. If $\mathcal{P}$ is closed, then $h_{0}=1$, and the denominator can be chosen in such a way that all $h_{i}$ are nonnegative. In the semiopen case such a representation may not exist. The theory of Ehrhart series is developed in several books, for example in [9]. For a treatment under the aspect of social choice we refer the reader to [23].

For closed polytopes $\mathcal{P}$, the Ehrhart function $E(\mathcal{P}, k)$ itself is given by a quasipolynomial $q_{\mathcal{P}}$ for all $k \geq 0$. Roughly speaking, this means that $q_{\mathcal{P}}(k)$ is a polynomial whose coefficients depend periodically on $k$. The period is a divisor of the least common multiple of the exponents $g$ in the factors $1-t^{g}$ in the denominator. In the Normaliz output the period is always exactly the least common multiple. In the semiopen case one has $E(\mathcal{P}, k)=q_{\mathcal{P}}(k)$ only for sufficiently large $k$. More precisely, if $r$ is the degree of $E_{\mathcal{P}}(t)$ as a rational function, then $E(\mathcal{P}, r) \neq q_{\mathcal{P}}(r)$, but $E(\mathcal{P}, k)=q_{\mathcal{P}}(k)$ for all $k>r$.

For $n=4$ the Ehrhart series of $\mathcal{C}$ has the numerator

$$
\begin{aligned}
& 6 t^{1}+15 t^{2}+481 t^{3}+890 t^{4}+12346 t^{5}+17845 t^{6} \\
+ & 152891 t^{7}+180850 t^{8}+1113216 t^{9}+1111974 t^{10}+5320122 t^{11} \\
+ & 4580485 t^{12}+17837843 t^{13}+13415068 t^{14}+43770180 t^{15}+28993857 t^{16} \\
+ & 80758791 t^{17}+47336170 t^{18}+113925878 t^{19}+59177761 t^{20}+123966919 t^{21} \\
& +56990048 t^{22}+104272000 t^{23}+42243510 t^{24}+67509138 t^{25}+23917200 t^{26} \\
& +33268048 t^{27}+10182887 t^{28}+12235441 t^{29}+3176870 t^{30}+3255226 t^{31} \\
& +697232 t^{32}+596834 t^{33}+100915 t^{34}+69821 t^{35}+8655 t^{36} \\
& +4581 t^{37}+363 t^{38}+133 t^{39}+5 t^{40}+t^{41} .
\end{aligned}
$$

The Ehrhart series of $\bar{\complement}$ has the numerator

$$
\begin{aligned}
& 1+5 t^{1}+133 t^{2}+363 t^{3}+4581 t^{4} \\
+ & 8655 t^{5}+69821 t^{6}+100915 t^{7}+596834 t^{8}+697232 t^{9} \\
+ & 3255226 t^{10}+3176870 t^{11}+12235441 t^{12}+10182887 t^{13}+33268048 t^{14}
\end{aligned}
$$




$$
\begin{aligned}
& +23917200 t^{15}+67509138 t^{16}+42243510 t^{17}+104272000 t^{18}+56990048 t^{19} \\
& +123966919 t^{20}+59177761 t^{21}+113925878 t^{22}+47336170 t^{23}+80758791 t^{24} \\
& +28993857 t^{25}+43770180 t^{26}+13415068 t^{27}+17837843 t^{28}+4580485 t^{29} \\
& +5320122 t^{30}+1111974 t^{31}+1113216 t^{32}+180850 t^{33}+152891 t^{34} \\
& +17845 t^{35}+12346 t^{36}+890 t^{37}+481 t^{38}+15 t^{39}+6 t^{40}
\end{aligned}
$$

Both have the same denominator

$$
(1-t)\left(1-t^{2}\right)^{14}\left(1-t^{4}\right)^{9}
$$

Numerator and denominator are coprime, but in general one cannot find a coprime representation if one insists on a denominator that is a product of terms $1-t^{g}$.

If we write the numerator of $\overline{\mathcal{C}}$ as $\sum_{i=0}^{40} a_{i} t^{i}$, then the numerator of $\mathcal{C}$ is $\sum_{i=0}^{40} a_{40-i} t^{i+1}$ : up to a shift in degree, they are palindromes of each other. This rather unexpected relationship is not an accident, and is explained by the following theorem.

Theorem 5. Let $\lambda_{1}, \ldots, \lambda_{m}$ be linear forms on $\mathbb{R}^{d}$, and let $\mathbf{1} \in \mathbb{R}^{d}$ be the vector with the entry 1 at all coordinates. Suppose that $\lambda_{i}(\mathbf{1})=0$ for all $i=1, \ldots, m$. Set $C=\left\{x \in \mathbb{R}_{+}^{d}\right.$ : $\left.\lambda_{i}(x) \geq 0, i=1 \ldots, m\right\}$, and

$$
D=\left\{x \in \mathbb{R}_{+}^{d}: \lambda_{i}(x)>0, i=1 \ldots, m\right\} .
$$

Define the he semiopen polytope $\mathcal{P}$ by

$$
\mathcal{P}=\left\{x \in D: \sum x_{i}=1\right\} .
$$

If $\operatorname{dim} \mathcal{P}=d-1$ (the maximal dimension), then the following hold:

(1) $J=I-1$, where $J$ is the set of lattice points in $D$ and I is the set of interior lattice points of $C$.

(3) Suppose that

$$
E_{\mathcal{P}}(t)=(-1)^{d} t^{-d} E_{\overline{\mathcal{P}}}\left(t^{-1}\right)
$$

$$
E_{\overline{\mathcal{P}}}(t)=\frac{h_{0}+h_{1} t \cdots+h_{s} t^{s}}{\left(1-t^{g_{1}}\right) \cdots\left(1-t^{g_{d}}\right)}
$$

Then the Ehrhart series of $\mathcal{P}$ has the numerator polynomial

$$
h_{s} t^{w}+\cdots+h_{0} t^{w+s}, \quad w=\sum_{i=1}^{d} g_{i}-d-s,
$$

over the same denominator.

Proof. The crucial observation is (1). Since $\operatorname{dim} \mathcal{P}=d-1$, the interior of $C$ is

$$
\left\{x: x_{i}>0, i=1, \ldots, d, \lambda_{j}(x)>0, j=1, \ldots, m\right\} .
$$

For lattice points $x \in \mathbb{Z}^{d}$ these inequalities amount to $x_{i} \geq 1$ and $\lambda_{j}(x) \geq 1$. Thus $x$ belongs to the interior of $C$ if and only if $x-1$ satisfies the inequalities $x_{i} \geq 0$ for $i=1, \ldots, d$, and $\lambda_{j}(x)>0$ for $j=1, \ldots, m$. This proves $J=I-\mathbf{1}$. 
By Ehrhart reciprocity (for example, see [9, Th. 6.51]) the Ehrhart series of the interior of $\overline{\mathcal{P}}$ is $(-1)^{d} E_{\overline{\mathcal{P}}}\left(t^{-1}\right)$. In view of (1) we have to multiply this series by $t^{-d}$ to obtain the Ehrhart series of $\mathcal{P}$. This gives (2).

Part (3) is now an elementary transformation.

Remark 6. (a) The condition $\lambda_{i}(\mathbf{1})=0$ in Theorem 5 is equivalent to the natural assumption that it only depends on the differences $v_{i}-v_{j}$ whether a voting result $\left(v_{1}, \ldots, v_{d}\right)$ belongs to the event defined by the inequalities.

(b) We have formulated Theorem 5 for the grading by total degree. It can easily be generalized to other gradings.

In the case of our polytopes we have $d=24$. So, for $\mathcal{C}$ we obtain $\sum_{i=1}^{d} g_{i}-d-s=$ $65-24-40=1$, as observed. Theorem 5 is applicable to all polytopes in Section 2 with the exception of $\mathcal{F}$. Nevertheless we have computed both Ehrhart series in each case since the comparison is an excellent test of the Normaliz algorithm. For $\mathcal{F}$ the formula in Theorem 5 does indeed not hold.

The Ehrhart quasipolynomials of $\mathcal{C}$ and $\overline{\mathcal{C}}$ have period 4. Moreover, they are equal for an odd number of voters $k$ and have the same expression

$$
\#\left(\mathcal{C}_{k} \cap \mathbb{Z}^{N}\right)=\#\left(\bar{\complement}_{k} \cap \mathbb{Z}^{N}\right)=\frac{R_{1,3}(k) *(12+k) * \prod_{i=1, i \text { odd }}^{23}(i+k)}{23 ! * 131072}
$$

(for $k \equiv 1,3 \bmod 4)$, where

$$
\begin{aligned}
R_{1,3}(k) & =261812975764725+308449567353120 k+165347938576012 k^{2} \\
& +50600971266720 k^{3}+9607752151310 k^{4}+1183838427360 k^{5} \\
& +96296973756 k^{6}+5130593760 k^{7}+172122725 k^{8}+3296640 k^{9}+27472 k^{10} .
\end{aligned}
$$

Let us reformulate this result in terms of probabilities. With the notation introduced at the beginning of Section 2, we have

$$
p_{\mathrm{CW}}^{(4)}(k)=4 p_{\mathrm{CW}}^{(4,1)}(k)
$$

where

Since

$$
p_{\mathrm{CW}}^{(4,1)} k(k)=\frac{\#\left(\mathcal{C}_{k} \cap \mathbb{Z}^{N}\right)}{\#\left(\mathcal{U}_{k} \cap \mathbb{Z}^{N}\right)} .
$$

we get

$$
\#\left(\mathcal{U}_{k} \cap \mathbb{Z}^{N}\right)=\frac{\prod_{i=1}^{23}(i+k)}{23 !}
$$

$$
p_{\mathrm{CW}}^{(4)}(k)=\frac{R_{1,3}(k) *(12+k)}{32768 * \prod_{i=1, i \text { even }}^{23}(i+k)} \quad \text { if } k \equiv 1,3 \bmod 4 .
$$

This is exactly the same formula as the one computed first by Gehrlein in [19]. For the case of even $k$ we set

$$
\begin{aligned}
R_{0}(k) & =4981367114669230129152000+11069309139290261311979520 k+11286725167650172468985856 k^{2} \\
& +6970525765323041332002816 k^{3}+2896901556002851225731072 k^{4}+857336679021412589010944 k^{5} \\
& +187293111169997407690752 k^{6}+30935327102400429176832 k^{7}+3923664152075008433664 k^{8}
\end{aligned}
$$




$$
\begin{aligned}
& +385511913998009006208 k^{9}+29422431828810359328 k^{10}+1738486466127164288 k^{11}+ \\
& +78715287099505056 k^{12}+2678620940814672 k^{13}+66260942646564 k^{14} \\
& +1124326347564 k^{15}+11698573833 k^{16}+56262656 k^{17}
\end{aligned}
$$

and

$$
\begin{aligned}
R_{2}(k) & =9794451243189989376000+921057250987916963020800 k+1705900639387417842032640 k^{2} \\
& +1489106767895973053595648 k^{3}+792353026020511342854144 k^{4}+284373446368099671547904 k^{5} \\
& +72772788665361422238720 k^{6}+13747699097527641501696 k^{7}+1960073323091557035648 k^{8} \\
& +213683286033339310848 k^{9}+17913763440866689440 k^{1} 0+1153396601212907264 k^{1} 1 \\
& +56538334354261872 k^{1} 2+2071748534241792 k^{1} 3+54936786331200 k^{1} 4 \\
& +995421043392 k^{1} 5+11023421961 k^{1} 6+56262656 k^{1} 7,
\end{aligned}
$$

then we get

$$
p_{\mathrm{CW}}^{(4)}(k)= \begin{cases}\frac{R_{0}(k) * k}{67108864 * \prod_{i=0}^{5}(1+4 * i+k)(2+4 * i+k)(3+4 * i+k)} & \text { if } k \equiv 0 \bmod 4 ; \\ \frac{R_{2}(k) * k}{67108864 * \prod_{i=0}^{5}(4 * i+k)(1+4 * i+k)(3+4 * i+k)} & \text { if } k \equiv 2 \bmod 4 .\end{cases}
$$

To the best of our knowledge the above formula for an even number of voters has not been computed before our computations with Normaliz.

Remark 7. With the notation used in [21, Formula 1.27 and 1.29], we have

$$
p_{\mathrm{CW}}^{(4)}(k)=P_{\mathrm{PMRW}}^{S}(4, k, \mathrm{IAC}) \quad \text { for all } k \in \mathbb{Z}_{+} .
$$

For the other eight polytopes, since the numerators of the Ehrhart series are very long, we only list the denominators for a representation similar to the one given above for $\mathcal{C}$ and $\overline{\mathrm{C}}$ (with non-coprime numerators):

$$
\begin{aligned}
\mathcal{Q}, \overline{\mathcal{Q}} & :(1-t)\left(1-t^{2}\right)^{2}\left(1-t^{4}\right)^{5}\left(1-t^{12}\right)^{16} \\
\mathcal{E}, \overline{\mathcal{E}}, \mathcal{F}, \overline{\mathcal{F}}, \mathcal{B}_{\mathrm{Sg}}, \overline{\mathcal{B}_{\mathrm{Sg}}}: & (1-t)\left(1-t^{2}\right)^{2}\left(1-t^{4}\right)^{5}\left(1-t^{12}\right)^{4}\left(1-t^{24}\right)\left(1-t^{120}\right)^{11}, \\
\mathcal{T}, \overline{\mathcal{T}}: & (1-t)\left(1-t^{2}\right)^{14}\left(1-t^{4}\right)^{5}\left(1-t^{12}\right)^{3}\left(1-t^{24}\right), \\
\mathcal{K}, \overline{\mathcal{K}}: & (1-t)\left(1-t^{2}\right)^{14}\left(1-t^{4}\right)^{5}\left(1-t^{12}\right)^{4} \\
\mathcal{B}_{\mathrm{St}}, \overline{\mathcal{B}_{\mathrm{St}}}: & (1-t)\left(1-t^{2}\right)^{2}\left(1-t^{4}\right)^{5}\left(1-t^{12}\right)^{4}\left(1-t^{24}\right)\left(1-t^{120}\right)^{4} \\
& \left(1-t^{840}\right)^{2}\left(1-t^{2520}\right)^{2}\left(1-t^{27720}\right)^{2}\left(1-t^{55440}\right), \\
\mathcal{B}_{\mathrm{SgRev}}, \overline{\mathcal{B}_{\mathrm{SgRev}}}: & (1-t)\left(1-t^{2}\right)^{2}\left(1-t^{4}\right)^{6}\left(1-t^{12}\right)^{3}\left(1-t^{24}\right)^{12}
\end{aligned}
$$

The denominators have 24 factors. All computed data is available and will be provided by the authors on request.

The reciprocity between $E_{\mathcal{P}}(t)$ and $E_{\overline{\mathcal{P}}}$ in Theorem 5 can be recast into a relation between the Ehrhart quasipolynomials. In terms of quasipolynomials, Ehrhart reciprocity says $q_{\text {relint } \overline{\mathcal{P}}}(k)=(-1)^{d-1} q_{\overline{\mathcal{P}}}(-k)$ for all $k \geq 1$ (for example, see [9, Th. 6.51]), and in view of Theorem 5 this implies

$$
q_{\mathcal{P}}(\ell)=(-1)^{d-1}(-\ell-d) .
$$


It follows that under the conditions of Theorem 5 one has $E(\mathcal{P}, k)=q_{\mathcal{P}}(k)$ for all $k>-d$, and therefore for all $k>0$.

Remark 8. (a) In Table 8 we summarize the essential data of the numerators of the Ehrhart series of the polytopes (with the exception of $\mathcal{F}$ ), according to the notations introduced in Theorem 5. The last column represents the period of the associated Ehrhart quasipolynomials.

\begin{tabular}{|r|r|r|r|r|r|}
\hline Polytope & $d$ & $s$ & $\sum g_{i}$ & $w$ & period \\
\hline $\mathcal{C}$ & 24 & 40 & 65 & 1 & 4 \\
\hline $\mathcal{Q}$ & 24 & 190 & 217 & 3 & 12 \\
\hline $\mathcal{E}$ & 24 & 1392 & 1417 & 1 & 120 \\
\hline $\mathcal{T}$ & 24 & 84 & 109 & 1 & 24 \\
\hline $\mathcal{K}$ & 24 & 70 & 97 & 3 & 12 \\
\hline $\mathcal{B}_{S t}$ & 24 & 118144 & 118177 & 9 & 55440 \\
\hline $\mathcal{B}_{S g}$ & 24 & 1388 & 1417 & 5 & 120 \\
\hline $\mathcal{B}_{\text {SgRev }}$ & 24 & 326 & 353 & 3 & 24 \\
\hline
\end{tabular}

TABLE 8. Data for the numerators of the Ehrhart series and quasipolynomials

(b) The numerator of $\overline{\mathcal{F}}$ is a polynomial of degree 1386 whereas the numerator of $\mathcal{F}$ has degree 1389. They are not related by Theorem 5, so the Ehrhart series must be computed separately by Normaliz. The Ehrhart quasipolynomials of $\mathcal{F}$ and $\overline{\mathcal{F}}$ have period 120 .

(c) The number $w$ in Table 8 is the smallest number of voters that can realize the respective election outcome since it is the lowest exponent of $t$ that appears in the Ehrhart series (when written as a power series). Note the different values of $\mathcal{B}_{S g}$ and $\mathcal{B}_{\mathrm{SgRev}}$ for $w$.

The values of $w$ can be checked by elementary arguments, independently from the Ehrhart series computation. We explain it for the strong Borda paradox. Let candidate 1 be the plurality winner. Then he has got more first places, say $m$, than any of the three other candidates. On the other hand, he is the Condorcet loser, and therefore there must be behind any of the other candidates in at least $m+1$ preference orders. Another candidate, for example 2, gets first place in at least one of these $m+1$ preference orders. Then $m>1$, so $m \geq 2$. For the total number of voters $k$ it follows that $k \geq 2 m+1 \geq 5$. But the strong Borda paradox can be realized by 5 voters: two of them place candidate 1 first, and the other three place the different other 3 candidates first, but candidate 1 last. Note that the same argument works for any number of candidates, with the exception of the three candidates situation where the minimal number of voters is 7 . Similarly one can see that, for four candidates elections, the reverse strong Borda paradox requires only 3 voters and the strict Borda paradox needs 9 of them.

\section{THE EXPLOITATION OF SYMMETRY}

The elegant approach of Schürmann in [24] for the computation of the volumes of $\mathcal{C}$ and variants of $\mathcal{Q}$ and $\mathcal{E}$ uses the high degree of symmetries of these polytopes. Suppose 
that a polytope $P \subset \mathbb{R}^{d}$ is defined by the sign inequalities $x_{i} \geq 0, i=1, \ldots, d$, and further inequalities $\lambda_{i}(x) \geq 0$ (or $>0$ ). If certain variables $x_{i_{1}}, \ldots, x_{i_{u}}$ occur in all of the linear forms $\lambda_{i}$ with the same coefficient (that may depend on $i$ ), then any permutation of them acts as a symmetry on the corresponding polytope, and the variables $x_{i_{1}}, \ldots, x_{i_{u}}$ can be replaced by their sum $y_{j}=x_{i_{1}}+\cdots+x_{i_{u}}$ in the $\lambda_{i}$. (The polytopes may have further symmetries.) The substitution can be used for a projection into a space of much lower dimension, mapping the polytope $P$ to a polytope $Q$ (this requires that the grading affine hyperplane $A_{1}$ is mapped onto an affine hyperplane by the projection). Instead of counting the lattice points in $k P$ one counts the lattice points in $k Q$ weighted with their number of preimage lattice points in $k P$. This amounts to the consideration of a weighted Ehrhart function

$$
k \mapsto \sum_{y \in k Q \cap \mathbb{Z}^{d}} f(y) .
$$

The polynomial $f$ is easily determined by elementary combinatorics: if $y_{j}, j=1, \ldots, m$, is the sum of $u_{j}$ variables $x_{i}$, then

$$
f(y)=\left(\begin{array}{c}
u_{1}+y_{1}-1 \\
u_{1}-1
\end{array}\right) \cdots\left(\begin{array}{c}
u_{m}+y_{m}-1 \\
u_{m}-1
\end{array}\right) .
$$

The theory of weighted Ehrhart functions has recently been developed in several papers; see Baldoni et al. [5], [6], [15]. In [24], only the leading form of the polynomial $f$ is used. Integration of the leading form with respect to Lesbesgue measure yields the volume.

In the case of the Condorcet polytope $\mathcal{C}$ for four candidates the symmetrization yields a polytope of dimension 7: there are two groups of 6 variables each that can be replaced by their sums, and 6 groups of two variables each. We leave it to the reader to spot them. Fortunately Normaliz finds them automatically. The polynomial $f$, also computed automatically, is

$$
f(y)=\left(\begin{array}{c}
y_{1}+5 \\
5
\end{array}\right)\left(y_{2}+1\right)\left(y_{3}+1\right)\left(y_{4}+1\right)\left(y_{5}+1\right)\left(y_{6}+1\right)\left(y_{7}+1\right)\left(\begin{array}{c}
y_{8}+5 \\
5
\end{array}\right)
$$

in this case. Among our polytopes, only $\mathcal{T}$ and $\mathcal{B}_{\text {St }}$ do not allow any symmetrization.

Version 3.2.1 of Normaliz calls its offspring NmzIntegrate behind the scenes for the symmetrized computation of volumes and weighted Ehrhart series. In version 3.3.0, NmzIntgerate will be included in Normaliz itself and no longer exist as a separate program. The algorithmic approach of NmzIntegrate is developed in [15]. For polynomial arithmetic Normaliz uses CoCoALib by Abbott, Bigatti and Lagorio [1].

\section{COMPUTATIONAL REPORT}

In this section we want to document the use of Normaliz 3.2.1 and computations performed with it during the preparation of this work.

5.1. Use of Normaliz. Normaliz is distributed as open source under the GPL. In addition to the source code, the distribution contains executables for the major platforms Linux, Mac and Windows. We include some details on the use of Normaliz in order to show that 
the input files have a transparent structure and that the syntax of the execution command is likewise simple.

The polytope $\mathcal{F}$ has the following input file:

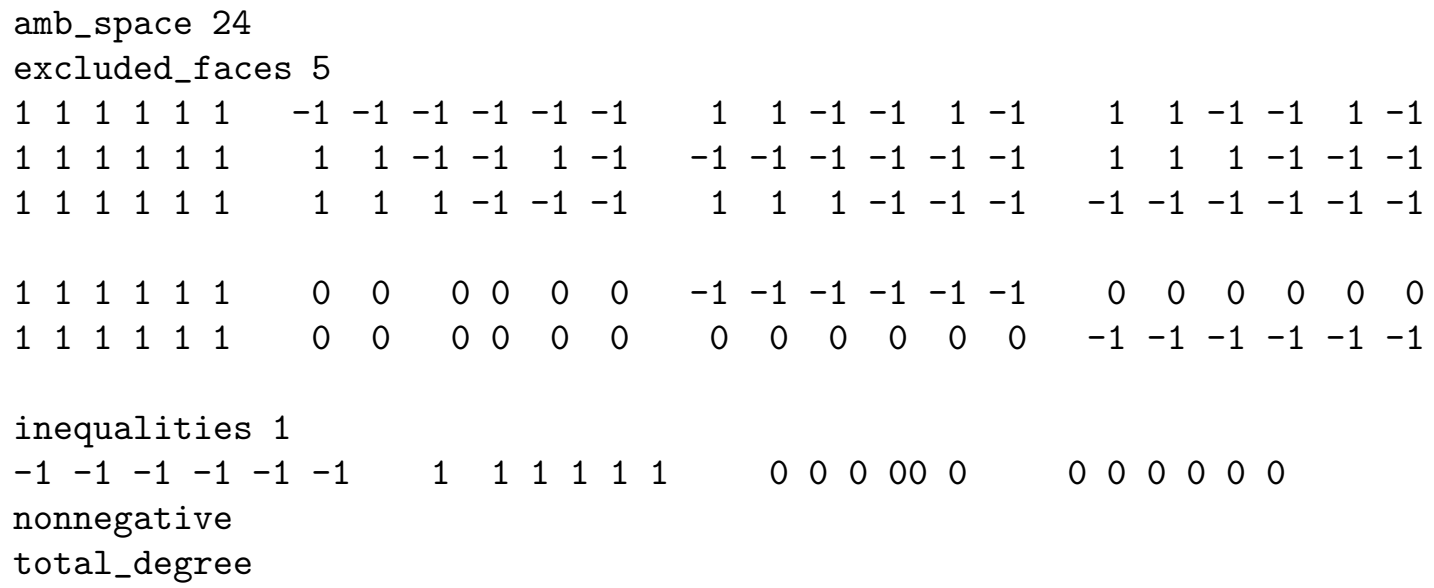

The first line amb_space 24 sets the ambient space to $\mathbb{R}^{24}$. The 5 excluded_faces represent the strict inequalities $\lambda_{i}>0, i=1,2,3,5,6$ of Table 3 , whereas the non-strict inequality $\overline{-\lambda_{4}}(v) \geq 0$ is given by 1 inequalities. The keyword nonnegative indicates that all 24 coordinates are to be taken nonnegative, whereas total_degree defines the grading in which each coordinate has weight 1 . Let us suppose the file is called CEP. in.

For Normaliz 3.2.1 and newer versions the simplest command for the computation is normaliz CEP

Depending on the installation, it may be necessary to prefix normaliz or CEP by a path. Often one adds the option - $c$ to get terminal output showing the progress of the computation. If no further option is added, Normaliz will compute the Hilbert series and the Hilbert basis. The computation of the latter is very fast in all cases of this paper, but one can suppress it by the option -q. If one is only interested in the volume, $-v$ is the right choice. The number of parallel threads can be limited via the option $-\mathrm{x}=\langle\mathrm{N}\rangle$ where $\langle\mathrm{N}\rangle$ stands for the number of threads. The computation results are contained in CEP. out.

As will be apparent from the terminal output (obtained with -c), Normaliz successfully tries symmetrization, and employs its companion NmzIntegrate as mentioned out above. One should note that Normaliz does automatic symmetrization only if the cone $D$ defined by image $\mathcal{Q}$ has dimension $\leq 2 \operatorname{dim}(C) / 3$ where $C$ is the cone over the polytope $\mathcal{P}$ to be computed. The bound has been introduced since one cannot expect a saving in computation time if the dimension does not drop enough. However, the user can force Normaliz to use symmetrization.

Remark 9. (a) Normaliz has an input type strict_inequalities. While it seems a natural choice and will yield the same results as excluded_faces, its use for the computations of this paper is not advisable since the algorithmic approach does not (yet) allow symmetrization, and even for cases without symmetrization it is usually significantly slower than excluded_faces.

(b) A graphical interface called jNormaliz (Almendra and Ichim [2]) is also available in the Normaliz package. For its use Java must be installed on the system. 
5.2. Overview of the examples. The columns of Table 9 contain the values of characteristic numerical data of the examples studied, namely: \#vertices is the number of the vertices of the polytope, and \#supp the number of its support hyperplanes. \#Hilb is the size of the Hilbert basis of the Ehrhart cone over the polytope (see [11] for more details). These data are invariants of the polytope.

\begin{tabular}{|r|r|r|r|r|r|}
\hline Polytope & \#vertices & \#supp & \#Hilb & \# triangulation & \# Stanley dec \\
\hline $\mathcal{C}$ & 234 & 27 & 242 & $1,344,671$ & $1,816,323$ \\
\hline $\mathcal{2}$ & 2418 & 28 & 12,948 & $343,466,918,256$ & $2,217,999,266,634$ \\
\hline $\mathcal{E}$ & 4644 & 30 & 31,308 & $464,754,352,804$ & $1,661,651,089,155$ \\
\hline $\mathcal{F}$ & 4572 & 30 & 26,325 & $1,009,992,718,827$ & $3,400,149,589,030$ \\
\hline $\mathcal{T}$ & 491 & 30 & 546 & $2,852,958$ & $5,635,927$ \\
\hline $\mathcal{K}$ & 262 & 28 & 362 & $1,346,894$ & $2,694,560$ \\
\hline $\mathcal{B}_{\mathrm{St}}$ & 6363 & 33 & 21,137 & $30,399,162,846$ & $75,933,588,203$ \\
\hline $\mathcal{B}_{\mathrm{Sg}}$ & 3216 & 30 & 24,816 & $149,924,230,551$ & $858,660,657,413$ \\
\hline $\mathcal{B}_{\mathrm{SgRev}}$ & 3432 & 30 & 9,548 & $366,864,865,269$ & $1,141,025,866,136$ \\
\hline
\end{tabular}

TABLE 9. Numerical data of test examples

The last two columns list the number of simplicial cones in the triangulation and the number of components of the Stanley decomposition (see [13] for details on these numbers). These data are not invariants of the polytope. The information is included to show the complexity of the computations if symmetrization is not used. Normaliz can do all computations without symmetrization, but then some of them will take days, even those with a high degree of symmetry. The size of the lexicographic triangulation depends on the order in which the extreme rays are processed. The polytopes in the table above are defined by their support hyperplanes, and therefore Normaliz first computes the extreme rays from them. The order used in the computations mentioned in Table 9 is not necessarily identical with the order produced by previous versions of Normaliz. Moreover, bottom decomposition, see [16], is used automatically if the ratio of the largest degree among the generators and the smallest is $\geq 10$. This further influences the data contained in the last two columns.

Of all our polytopes, only $\mathcal{T}$ and $\mathcal{B}_{\mathrm{St}}$ cannot be symmetrized. The combinatorial data of the symmetrized polytopes are contained in Table 10 .

We remark that, for Hilbert basis computations, the dual algorithm of Normaliz (see [11]) is much faster than the primal algorithm for the examples of this paper, and all computations run in a few seconds. This is by no means always the case (see [13]).

5.3. Hardware characteristics. Almost all computations were run on a compute server with operating system CentOS 7.3, 4 Intel Xeon E5-2660 at 2.20GHz (a total of 32 cores) and $192 \mathrm{~GB}$ of RAM. With the exception of $\mathcal{B}_{\mathrm{St}}$, all computations were also done on a standard laptop with operating system Ubuntu 16.04, an Intel i5-4200M CPU at 2.5 Ghz and 12 GB RAM. In parallelized computations we have limited the number of threads used to 30. In Tables 11 and 13, 4x and 30x indicates parallelization with 4 and 30 threads, respectively. As the large examples below show, the parallelization scales efficiently; see 


\begin{tabular}{|r|r|r|r|r|r|}
\hline Polytope & dim & \#vertices & \#supp & \# triangulation & \# Stanley dec \\
\hline $\mathcal{C}$ & 8 & 16 & 11 & 17 & 33 \\
\hline $\mathcal{Q}$ & 6 & 12 & 8 & 14 & 14 \\
\hline $\mathcal{E}$ & 13 & 170 & 18 & 18208 & 19999 \\
\hline $\mathcal{F}$ & 13 & 163 & 18 & 23738 & 41963 \\
\hline $\mathcal{K}$ & 14 & 63 & 18 & 1035 & 2070 \\
\hline $\mathcal{B}_{\text {Sg }}$ & 13 & 100 & 18 & 3696 & 6025 \\
\hline $\mathcal{B}_{\text {SgRev }}$ & 13 & 115 & 19 & 10342 & 26024 \\
\hline
\end{tabular}

TABLE 10. Numerical data of symmetrized polytopes

also Table 12. The version that we have used exchanges data via files. The laptop has an SSD , but the server has only hard disks, and it is not a local hard disk of the machine, but the files must go through the NFS, the network file system.

Normaliz and NmzIntegrate need relatively little memory. All computations mentioned in this paper run stably with $<0.5 \mathrm{~GB}$ of RAM for each thread used.

5.4. Volumes. Table 11 contains the computation times for the volumes of the studied polytopes. The input for all these examples is given in the form of inequalities. When one runs Normaliz on such examples, it first computes the extreme rays of the cone and uses them as generators. This small extra time is also included in the reported times below. (It is also apparent and not surprising that small examples profit from a small number of parallel threads.)

\begin{tabular}{|r|c|r|r|}
\hline Polytope & Symmetrize & Laptop 4x & Server 30x \\
\hline $\mathcal{C}$ & Yes & $0.100 \mathrm{~s}$ & $0.591 \mathrm{~s}$ \\
\hline $\mathcal{Q}$ & Yes & $0.33 \mathrm{~s}$ & $0.76 \mathrm{~s}$ \\
\hline $\mathcal{E}$ & Yes & $1: 11: 39 \mathrm{~h}$ & $8: 41 \mathrm{~m}$ \\
\hline $\mathcal{F}$ & Yes & $1: 48: 57 \mathrm{~h}$ & $15: 06 \mathrm{~m}$ \\
\hline $\mathcal{T}$ & No & $7.200 \mathrm{~s}$ & $10.455 \mathrm{~s}$ \\
\hline $\mathcal{K}$ & Yes & $0.660 \mathrm{~s}$ & $1.940 \mathrm{~s}$ \\
\hline $\mathcal{B}_{\mathrm{St}}$ & No & - & $3: 57: 26 \mathrm{~h}$ \\
\hline $\mathcal{B}_{\mathrm{Sg}}$ & Yes & $14: 51 \mathrm{~m}$ & $1: 39 \mathrm{~m}$ \\
\hline $\mathcal{B}_{\operatorname{SgRev}}$ & Yes & $44: 54 \mathrm{~m}$ & $4: 17 \mathrm{~m}$ \\
\hline
\end{tabular}

TABLE 11. Computation times for volumes

In order to measure the parallelization we have run the volume computation of $\mathcal{E}$ with varying number of threads. Table 12 shows that NmzIntegrate is very efficiently parallelized.

Remark 10. The volume of the polytope $\mathcal{C}$ was first computed by Gehrlein [19]. The volumes of variants of the polytopes $\mathcal{Q}$ and $\mathcal{E}$ had been computed by Schürmann [24] with LattE integrale [4]. This information was very useful for checking the correctness of Normaliz. 


\begin{tabular}{|c||r|r|r|r|r|}
\hline \# threads & 1 & 5 & 10 & 20 & 30 \\
\hline real time s & 10367 & 2380 & 1245 & 656 & 521 \\
\hline efficiency \% & 100 & 88 & 83 & 79 & 66 \\
\hline
\end{tabular}

TABLE 12. Efficiency of parallelization in volume computations

5.5. Ehrhart series and quasipolynomials. The experimental times obtained for computation of Ehrhart series and quasipolynomials are contained in Table 13. As above, the presented times include the time used by Normaliz for computing the extreme rays of the cone. Moreover, the Ehrhart quasipolynomials are computed from the Ehrhart series (see [11]). This requires for some examples like $\mathcal{B}_{\mathrm{St}}$ a significant extra time, which has likewise been included. We have also measured the parallelization for the Ehrhart series computation of $\mathcal{B}_{\mathrm{St}}$; see Table 14. Somewhat surprisingly, the efficiency is $>100 \%$ for certain numbers of threads, an effect that can only be explained by the memory management of the system.

\begin{tabular}{|r|r|r|r|r|r|}
\hline Polytope & \multirow{2}{*}{ Symmetrize } & \multicolumn{2}{|c|}{ Laptop $4 \mathrm{x}$} & \multicolumn{2}{c|}{ Server 30x } \\
\cline { 3 - 6 } & & closed & semi-open & closed & semi-open \\
\hline $\mathcal{C}$ & Yes & $1.730 \mathrm{~s}$ & $1.940 \mathrm{~s}$ & $1.925 \mathrm{~s}$ & $2.077 \mathrm{~s}$ \\
\hline $\mathcal{Q}$ & Yes & $4.400 \mathrm{~s}$ & $7.64 \mathrm{~s}$ & $7.010 \mathrm{~s}$ & $8.440 \mathrm{~s}$ \\
\hline $\mathcal{E}$ & Yes & $4: 50: 55 \mathrm{~h}$ & $4: 45: 24 \mathrm{~h}$ & $28: 36 \mathrm{~m}$ & $41: 01 \mathrm{~m}$ \\
\hline $\mathcal{F}$ & Yes & $12: 02: 42 \mathrm{~h}$ & $12: 47: 03 \mathrm{~h}$ & $1: 45: 15 \mathrm{~h}$ & $1: 39: 19 \mathrm{~h}$ \\
\hline $\mathcal{T}$ & No & $16.230 \mathrm{~s}$ & $28.260 \mathrm{~s}$ & $24.050 \mathrm{~s}$ & $34.136 \mathrm{~s}$ \\
\hline $\mathcal{K}$ & Yes & $16.770 \mathrm{~s}$ & $25.810 \mathrm{~s}$ & $3.156 \mathrm{~s}$ & $7.967 \mathrm{~s}$ \\
\hline $\mathcal{B}_{\mathrm{St}}$ & No & - & - & $10: 08: 50 \mathrm{~h}$ & $37: 03: 26 \mathrm{~h}$ \\
\hline $\mathcal{B}_{\mathrm{Sg}}$ & Yes & $1: 34: 23 \mathrm{~h}$ & $1: 36: 16 \mathrm{~h}$ & $9: 01 \mathrm{~m}$ & $14: 13 \mathrm{~m}$ \\
\hline $\mathcal{B}_{\mathrm{SgRev}}$ & Yes & $5: 56: 18 \mathrm{~h}$ & $5: 53: 38 \mathrm{~h}$ & $45: 13 \mathrm{~m}$ & $47: 22 \mathrm{~m}$ \\
\hline
\end{tabular}

TABLE 13. Computation times for Ehrhart series and quasipolynomials

\begin{tabular}{|c||r|r|r|r|r|}
\hline \# threads & 1 & 5 & 10 & 20 & 30 \\
\hline real time s & 15959 & 398 & 1230 & 635 & 553 \\
\hline efficiency \% & 100 & 94 & 130 & 126 & 96 \\
\hline
\end{tabular}

TABLE 14. Efficiency of parallelization in Ehrhart series computations

\section{ACKNOWLEDGEMENT}

The authors like to thank Achill Schürmann for several test examples that were used during the development of Normaliz and NmzIntegrate. 


\section{REFERENCES}

[1] J. Abbott, A.M. Bigatti and G. Lagorio, CoCoA-5: a system for doing Computations in Commutative Algebra. Available at http://cocoa.dima.unige.it

[2] V. Almendra and B. Ichim: jNormaliz. A graphical interface for Normaliz. Available at www.math.uos.de/normaliz.

[3] D. Avis, lrs: A revised implementation of the reverse search vertex enumeration algorithm. Available at http://cgm.cs.mcgill.ca/ avis/C/lrs.html.

[4] V. Baldoni, N. Berline, J.A. De Loera, B. Dutra, M. Köppe, S. Moreinis, G. Pinto, M. Vergne, J. Wu, A User's Guide for LattE integrale v1.7.2, 2013. Software package LattE is available at http://www. math.ucdavis.edu/ latte/

[5] V. Baldoni, N. Berline, J.A. De Loera, M. Köppe and M. Vergne, How to integrate a polynomial over a simplex. Math. Comp. 80 (2011), 297-325.

[6] V. Baldoni, N. Berline, J.A. De Loera, M. Köppe and M. Vergne, Computation of the highest coefficients of weighted Ehrhart quasi-polynomials of rational polyhedra. Found. Comp. Math., in press.

[7] J.-C. Chevalier de Borda, Mémoire sur les élections au scrutin. Histoire de'Académie Royale Des Sci. 102 (1781), $657-665$.

[8] F. Brandt, C. Geist and M. Strobel, Analyzing the Practical Relevance of Voting Paradoxes via Ehrhart Theory, Computer Simulations, and Empirical Data. Proceedings of the 2016 International Conference on Autonomous Agents \& Multiagent Systems, 385-393.

[9] W. Bruns and J. Gubeladze, Polytopes, rings and K-theory, Springer, 2009.

[10] W. Bruns, R. Hemmecke, B. Ichim, M. Köppe, and C. Söger, Challenging computations of Hilbert bases of cones associated with algebraic statistics. Exp. Math. 20 (2011), 25-33.

[11] W. Bruns and B. Ichim, Normaliz: algorithms for affine monoids and rational cones. J. Algebra 324 (2010), 1098-1113.

[12] W. Bruns, B. Ichim, T. Römer, R. Sieg and C. Söger: Normaliz. Algorithms for rational cones and affine monoids. Available at http://normaliz.uos.de.

[13] W. Bruns, B. Ichim and C. Söger, The power of pyramid decomposition in Normaliz. Journal of Symbolic Computation 74 (2016), 513 - 536.

[14] W. Bruns and R. Koch, Computing the integral closure of an affine semigroup. Univ. Iagel. Acta Math. 39 (2001), 59-70.

[15] W. Bruns and C. Söger, Generalized Ehrhart series and Integration in Normaliz. J. Symb. Comp. 68 (2015), 75-86.

[16] W. Bruns, R. Sieg and C. Söger, Normaliz, 2013-2016. Preprint arXiv:1611.07965.

[17] N. Marquis de Condorcet Éssai sur l'application de l'analyse à la probabilité des décisions rendues à la pluralité des voix. Imprimerie Royale, Paris (1785).

[18] J.A. De Loera, B. Dutra, M. Köppe, S. Moreinis, G. Pinto and J. Wu, Software for exact integration of polynomials over polyhedra. Computational Geometry 46 (2013), 232-252.

[19] W.V. Gehrlein, Condorcet winners on four candidates with anonymous voters. Economics Letters 71 (2001), 335-340.

[20] W.V. Gehrlein and P. Fishburn, Condorcet's paradox and anonymous preference profiles. Public Choice 26 (1976), 1-18.

[21] W.V. Gehrlein and D. Lepelley, Voting Paradoxes and Group Coherence, Springer, 2011.

[22] W.V. Gehrlein and D. Lepelley, On the probability of observing Borda's paradox. Social Choice and Welfare 35 (2010), 1-23.

[23] D. Lepelley, A. Louichi and H. Smaoui, On Ehrhart polynomials and probability calculations in voting theory. Social Choice and Welfare 30 (2008), 363-383.

[24] A. Schürmann, Exploiting polyhedral symmetries in social choice. Social Choice and Welfare 40 (2013), 1097-1110.

[25] M.C. Wilson and G. Pritchard, Probability calculations under the IAC hypothesis. Math. Social Sci. 54 (2007), 244-256. 


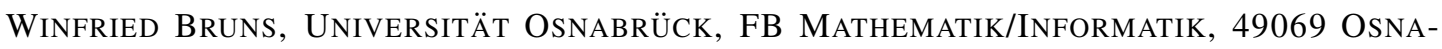
BRÜCK, GERMANY

E-mail address: wbruns@uos.de

Bogdan Ichim, Institute of Mathematics "Simion Stollow" of the Romanian ACADEMY, C.P. 1-764, 010702 BUCHAREST, ROMANIA

E-mail address: bogdan.ichim@imar.ro

Christof SöGer, Universität OSNabrüCK, FB Mathematik/INFormatik, 49069 OSNABRÜCK, GERMANY

E-mail address: csoeger@uos.de 